\title{
Cárie precoce da infância: fatores de risco associados
}

Early childhood caries: associated risk factors

\author{
Carie precoces de la infancia: factores de riesgo asociados \\ Maria das Graças Barbosa da SILVA 1 \\ Maria Helena Chaves de Vasconcelos CATÃO2 2 \\ Francisco Juliherme Pires de ANDRADRE ${ }^{3}$ \\ Catarina Ribeiro Barros de ALENCAR ${ }^{\mathbf{4}}$ \\ ${ }^{1}$ Mestranda do Programa de Pós-Graduação em Odontologia, Departamento de Odontologia, \\ Universidade Estadual da Paraíba, UEPB, 58429-500, Campina Grande - PB, Brasil \\ ${ }^{2}$ Professora Doutora do Programa de Pós-Graduação em Odontologia, Departamento de Odontologia, \\ Universidade Estadual da Paraíba, UEPB, 58429-500, Campina Grande - PB, Brasil \\ ${ }^{3}$ Professor Doutor em Odontologia, Departamento de Odontologia, Universidade Estadual da Paraíba, \\ UEPB, 58429-500, Campina Grande - PB, Brasil \\ ${ }^{4}$ Pós-Doutoranda do Programa de Pós-Graduação em Odontologia, Departamento de Odontologia \\ Universidade Estadual da Paraíba, UEPB, 58429-500, Campina Grande - PB, Brasil
}

\begin{abstract}
Resumo
Introdução: A etiologia clássica da cárie dentária é embasada na interação de fatores como dente suscetível, microrganismos, dieta e tempo que são influenciadas pela interação de múltiplos componentes sociais e ambientais, permitindo, assim, uma compreensão ainda mais fidedigna das situações de risco que permeiam o surgimento e desenvolvimento das lesões de cárie. Objetivo: Revisar a literatura acerca dos principais fatores de risco envolvidos no surgimento e progressão da cárie precoce da infância. Materiais e Métodos: A revisão de literatura foi conduzida nas bases de dados MEDLINE - PubMed, Scopus e Web of Science, abrangendo o período entre 2011 a 2017. Os termos utilizados foram: "risk factors" e "early childhood caries". Resultados: Verificou-se que os principais fatores de risco associados com a cárie precoce da infância foram: crianças mais velhas; baixo nível de escolaridade dos principais cuidadores; baixa renda familiar; habitantes de zona rural; escasso acesso a serviços de saúde bucal; hábitos alimentares inadequados; conhecimento limitado dos cuidadores sobre higiene bucal; idade tardia de início da escovação; não participação dos cuidadores nas escovações e comprometimento sistêmico. Conclusão: Considerando que todos os fatores de risco encontram-se em íntima relação, não é possivel avaliar quais, dentre os fatores associados, exercem maior ou menor influência no surgimento da cárie precoce da infância. Nesse sentido, o conhecimento sobre os fatores do risco associados permite uma atuação odontopediátrica centrada na prevenção dos agravos à saúde bucal.
\end{abstract}

Descritores: Cárie Dentária; Fatores de Risco; Odontopediatria.

\begin{abstract}
Introduction: The classic etiology of dental caries is based on the interaction of factors such as susceptible teeth, microorganisms, diet and time. They are influenced by the interaction of social and environmental componentes, thus allowing an even more reliable understanding of the risk situations that arise and development of caries lesions.Objective: To review the literature on the main risk factors involved in the onset and progression of early childhood caries. Materials and Methods: The literature review was conducted in the MEDLINE - PubMed, Scopus and Web of Science databases, covering the period between 2011 and 2017. The terms used were: "risk factors" and "early childhood caries." Results: It was found that the main risk factors associated with early childhood caries were: older children, low level of schooling of primary caregivers, low family income, rural inhabitants, poor access to oral health services, inadequate eating habits, limited knowledge of caregivers about oral hygiene, late age of onset of toothbrushing, lack of caregivers' participation in brushing and systemic impairment Conclusion: Considering that all risk factors are closely related, it is not possible to evaluate which, among the associated factors, exert greater or the early onset of early childhood caries. In this sense, knowledge about ssociates allows a pediatric dentistry centered on the prevention of oral health problems.
\end{abstract}

Descriptors: Dental Caries; Risk Factors; Pediatric Dentistry.

\section{Resumen}

La etiología clásica de la caries dental se basa en la interacción de factores como el diente susceptible, los microorganismos, la dieta y el tiempo influenciados por la interacción de múltiples componentes sociales y ambientales, permitiendo así una comprensión aún más fidedigna de las situaciones de riesgo que permean el surgimiento y el desarrollo de las lesiones de caries. Objetivo: Revisar la literatura acerca de los principales factores de riesgo involucrados en el surgimiento y progresión de la caries precoz de la infancia. Materiales y Métodos: La revisión de literatura se llevó a cabo en las bases de datos MEDLINE - PubMed, Scopus y Web of Science que abarca el período entre 2011 y 2017. Los términos utilizados fueron: "risk factors" y "early childhood caries" Resultados: Verificou-se que los principales factores de riesgo se asocian a un tratamiento previo de la infancia para: niños más velhas; bajo nivel de escolaridad de los principales cuidadores; familiar; habitantes de zona rural; escasso acceso a servicios de salud bucal; hábitos alimentares inadecuados; conocimiento limitado dos cuidadores sobre higiene bucal; tardía de inicio de la escovación; no participación de los cuidadores en las escobas y el compromiso sistémico. Conclusión: Considerando que todos los factores de riesgo se encuentran en una relación íntima, no existe un criterio de calidad, existe un mayor o menor impacto. Nesse sentido, el conocimiento sobre los riesgos del riesgo permite una actuación odontopediátrica centrada en la prevención de los agravos para el cuidado del salud bucal.

Descriptores: Caries Dental; Factores de Riesgo; Odontología Pediátrica.

\section{INTRODUÇÃO}

A cárie precoce da infância $(\mathrm{CPI})$ é definida pela American Academy of Pediatric Dentistry - Academia Americana de Odontopediatria como a presença de um ou mais dentes cariados (com lesões cavitadas ou não cavitadas), ausentes (devido à cárie) ou restaurados em crianças de até 71 meses de idade. Em crianças menores de três anos de idade, qualquer superfície dental lisa que esteja cariada (cavitada ou não), é indicativo de Cárie Severa da Infância. Também é considerada Cárie Severa da Infância se, dos 3 aos 5 anos de idade, a criança apresenta mais de quatro, cinco e seis superfícies afetadas em dentes anteriores decíduos aos 3, 4 e 5 anos de idade, respectivamente ${ }^{1}$.

A CPI é a doença crônica mais comum nos primeiros anos de vida e, atualmente, representa um grande problema para a saúde pública mundial, tanto em países industrializados quando em desenvolvimento, afetando uma considerável proporção de crianças pré-escolares que não se beneficiam de abordagens preventivas e onde os serviços de atenção à saúde são, muitas vezes, deficientes ${ }^{2-5}$.

No Brasil, os dados do último levantamento 
epidemiológico em saúde bucal demonstraram que, aos 5 anos de idade, 53,5\% das crianças brasileiras já apresentavam alguma experiência de cárie dentária. Embora seja possível observar variações nessa porcentagem ao se considerar as regiões geográficas estudadas, tendo sido encontrada prevalência de cárie de $58,4 \%$ na região nordeste, o valor médio do índice ceo-d (dentes decíduos cariados, perdidos e obturados) permite concluir que nesta idade as crianças brasileiras apresentam, em média, dois dentes cariados ${ }^{6}$

Especialmente em crianças pré-escolares, a cárie é uma condição potencialmente limitante. As sequelas podem incluir dor, comprometimento estético e psicossocial, dificuldades de sono e mastigação, fatores estes que são capazes de promover mudanças de comportamento e prejuízo no desempenho escolar. Podem-se observar ainda problemas de ordem sistêmica, como retardo no crescimento e baixo peso corporal, quando comparado a crianças livres de cárie, devido a uma provável ocorrência de dor durante a alimentação. Além disso, crianças com experiência de cárie na dentição decídua apresentam maior probabilidade de desenvolverem lesões de cárie na dentição permanente, impactando negativamente na qualidade de vida do indivíduo, com efeitos cumulativos ao longo dos $\operatorname{anos}^{7-11}$.

A cárie dentária é, contudo, uma doença evitável a partir do momento em que os fatores que levam a essa condição se tornam conhecidos. Trata-se de uma doença com etiologia multifatorial, cujos fatores de risco apresentam-se numa rede de causalidade, que considera a influência de fatores demográficos, socioeconômicos, comportamentais e biológicos ${ }^{12}$.

Desse modo, a partir do reconhecimento dos fatores de risco associados à manifestação da CPI, ações de prevenção e promoção de saúde podem ser planejadas no sentido de reorientar os saberes em relação ao desenvolvimento da doença cárie em crianças, na concepção de seus cuidadores.

Diante do exposto, o presente artigo objetiva revisar a literatura acerca dos principais fatores de risco envolvidos no surgimento e progressão da cárie precoce da infância.

\section{MATERIAL E MÉTODO}

A revisão da literatura foi conduzida nas bases de dados MEDLINE - PubMed, Scopus e Web of Science usando os termos: "risk factors" and "early childhood caries".

Os critérios de elegibilidade dos artigos foram os seguintes: artigos publicados na íntegra e com acesso livre e gratuito, ou controlado, do tipo pesquisa científica sobre o tema, no idioma inglês, português ou espanhol; publicados entre 2011 e 2017, além de artigos clássicos sobre a temática em estudo.

Foram encontrados 132 trabalhos indexados com os descritores selecionados, que foram avaliados mediante leitura do título e do resumo e disponibilidade para acesso ao trabalho na íntegra. Destes, 34 artigos cumpriram todos os critérios de elegibilidade e foram escolhidos para leitura completa e obtenção das informações apresentadas nesta revisão de literatura.

\section{REVISÃO DA LITERATURA}

A cárie dentária ocorre em virtude de uma mudança no equilíbrio homeostático da microbiota residente, devido a uma mudança nas condições ambientais locais que favorece o crescimento de bactérias cariogênicas ${ }^{13,14}$. Essas bactérias possuem a capacidade de metabolizar açúcar, produzindo ácidos, os quais, ao longo do tempo, irão promover a desmineralização da estrutura dental ${ }^{15}$.

O processo de desenvolvimento da lesão de cárie é o mesmo em dentes decíduos e permanentes, seja em esmalte ou dentina. Toda vez que açúcar é ingerido, as bactérias presentes no biofilme dentário produzem ácidos capazes de desmineralizar a estrutura mineral dos dentes durante 0 tempo em que o $\mathrm{pH}$ fica baixo $(<6,7$ para dentina e $<5,5$ para esmalte). Após certo tempo de exposição ao açúcar, o pH se eleva a valores acima dos críticos para o esmaltedentina e a saliva tende a repor os minerais dissolvidos, por meio de um fenômeno denominado remineralização ${ }^{16}$. Quando esse processo não é suficiente para conter a desmineralização da superfície dental, observa-se o surgimento de lesões brancas, as quais após sucessivos episódios de desmineralização ocasionam o aparecimento de cavidades e maior destruição do tecido da superfície dental $^{17}$.

A etiologia clássica da cárie é embasada na interação de fatores, como: dente suscetível, microrganismos e dieta, que são influenciadas pela interação de múltiplos fatores sociológicos e ambientais ${ }^{18}$. Assim, a prevalência da CPI e sua severidade não podem ser determinadas considerando apenas a relação direta de um único fator. Todos os fatores devem ser considerados e inter-relacionados, de modo a se atingir uma compreensão ainda mais fidedigna das situações que permeiam o meio onde a criança está inserida e interferem no surgimento das lesões de cárie ${ }^{19,20}$.

\section{- Fatores de risco associados à cárie precoce da infância}

\section{- Fatores Demográficos}

Os estudos apontam que a CPI é mais prevalente e severa em crianças mais velhas, observando que o maior índice de crianças livres cárie é encontrado entre as mais novas $^{21,22}$. Alguns estudos relatam que aos 4 anos de idade, a criança apresenta o maior índice de cárie, sendo que, aos 5 anos, no entanto, há uma diminuição nesse índice ${ }^{2,19}$

Uma relação estatisticamente significativa entre o sexo e a prevalência da CPI tem sido reportada, com predileção para indivíduos do sexo masculino ${ }^{2,23}$. Não obstante, alguns estudos relatam que não é possível traçar esta relação estatisticamente significante entre o sexo da criança e a presença da CPI ${ }^{19,23,24}$.

O local da residência, se em zona urbana ou zona rural, também exerce influência no desenvolvimento precoce de lesões de cáries, com o favorecimento das lesões em crianças residentes na zona rural ${ }^{19,20,24,25}$.

- Fatores Socioeconômicos

Estudos indicam que baixo nível socioeconômico constitui um fator de risco para a ocorrência de $\mathrm{CPI}^{26-29}$. Nesse sentido, os principais fatores associados são o grau de instrução dos cuidadores, a renda familiar mensal e o acesso a serviços de saúde.

O nível de escolaridade dos principais cuidadores, especialmente da mãe, representa um dos fatores mais significativos associado com a ocorrência e a severidade da cárie precoce em crianças $^{27,29}$.

No que tange à renda familiar mensal, observa-se que uma maior ocorrência de CPI é observada em crianças cujas famílias apresentam menores rendas salariais ${ }^{24,27,29-32}$.

$\mathrm{O}$ acesso precoce aos serviços de saúde bucal pode ser um fator protetor contra o desenvolvimento de lesões de cáries precoces, desde que a primeira consulta não seja motivada por episódios de dor ${ }^{24,26,28,30}$.

- Fatores Comportamentais 
De acordo com Chaffee et al. $^{32}$ o aleitamento materno superior a 24 meses está associado com o aumento da prevalência de CPI. Outros relatam que um tempo de aleitamento materno superior a 12 meses já pode ser considerado fator de risco para a $\mathrm{CPI}^{24,33}$. Nesse sentido, os casos mais severos de CPI são observados em crianças cujo aleitamento materno foi superior a 12 meses e uso de mamadeira superior há 2 anos ${ }^{19}$.

Estudos indicam que a amamentação noturna, seja esta por aleitamento materno ou artificial, em livre demanda, sem a devida higienização posterior, apresenta-se como um dos principais fatores de risco para o surgimento de lesões cariosas em crianças ${ }^{19,20,23,29}$.

Observa-se, também, que o consumo de doce, acima de duas vezes ao dia, aumenta consideravelmente a probabilidade de ocorrência de $\mathrm{CPI}^{3,17,19}$. Outrossim, o consumo de lanches entre as principais refeições também configura fator de risco para a CPI e aparece como uma prática presente em crianças com lesões de cárie ${ }^{22,34,35}$.

Há também consistentes associações entre o uso de chupetas com açúcar ou mel e o consumo de alimentos e

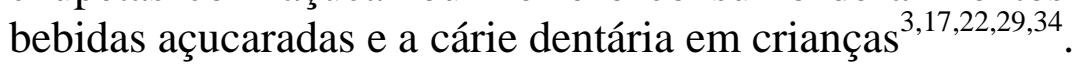

O conhecimento dos cuidadores sobre hábitos de higiene bucal é um fator relevante para a proteção das crianças contra a cárie dentária ${ }^{2}$, com destaque para o conhecimento sobre a idade de início da escovação ${ }^{22,26,33,34}$. A escovação dos dentes das crianças deve ser instituída logo quando da erupção do primeiro dente, já que as pesquisas apontam que crianças que começaram a escovar os dentes tardiamente apresentaram maior prevalência de cárie precoce na infância ${ }^{22,26,33,34}$

Além da idade de início da escovação, a supervisão dos cuidadores durante a escovação e escovações múltiplas tem grande importância ${ }^{19,34}$. Crianças menores não possuem habilidades manuais plenamente desenvolvidas, fazendo com que a escovação dos dentes, realizada por elas próprias, seja feita de maneira inadequada, uma vez que a eficácia da escovação dental é dependente da frequência, da técnica utilizada e do tempo gasto na escovação ${ }^{36}$.

A AAPD ${ }^{1}$ recomenda o uso de dentifrício fluoretado (na concentração mínima de 1.000 ppm F) duas vezes por dia desde a erupção do primeiro dente decíduo e a literatura tem evidenciado que crianças que usam dentifrício fluoretado possuem prevalência de cárie significativamente mais baixa ${ }^{37}$.

\section{- Fatores Biológicos}

A AAPD $^{1}$ prevê que as crianças com condições crônicas de saúde ou aquelas que fazem tratamento com medicamentos que alteram a composição e o fluxo salivar podem apresentar um maior risco de cárie dentária em relação a crianças sistemicamente saudáveis. Um estudo com 120 crianças chilenas, entre 2 e 5 anos de idade, avaliou a presença de CPI entre crianças consideradas saudáveis e crianças com doenças respiratórias crônicas. $\mathrm{O}$ resultado observado foi de que a CPI estava presente em $23 \%$ das crianças ditas saudáveis e em $55 \%$ das crianças enfermas ${ }^{15}$.

Crianças que nasceram abaixo do peso ideal ou que nasceram prematuras são predispostas a ter maiores níveis de colonização por Strepctococcus, defeitos de desenvolvimento de esmalte, que podem representar nichos para acúmulo de biofilme e desordens salivares, que comprometem as defesas naturais do organismo ${ }^{22,38}$. Além disso, crianças que se encontram abaixo ou acima do peso ideal possuem maiores índices de $\mathrm{CPI}^{24,25}$.

\section{DISCUSSÃO}

A cárie precoce da infância é uma doença de etiologia multifatorial e crônica, porém pode ser prevenida a partir do conhecimento dos fatores de risco para o seu surgimento e que conduzem a diversos agravos para a saúde da criança. Assim, crianças de até cinco anos de idade devem ser consideradas como grupo vulnerável e importante, uma vez que nesta faixa etária estão expostas às interferências diretas dos cuidadores e, ainda, se constroem os hábitos de dieta e higiene bucal que poderão perdurar no futuro da criança ${ }^{17}$.

Os estudos apontam que há uma relação diretamente proporcional entre o aumento da idade das crianças e uma maior prevalência de cárie dentária ${ }^{2,17,19,22-24}$ o que pode ser explicado pelo número crescente de dentes erupcionados, que ficam expostos ao meio bucal e ao desafio cariogênico e, também em decorrência de uma mudança nos hábitos alimentares e nas práticas de higiene bucal que impulsionam, cada vez mais, para a autonomia da criança em uma fase em que a destreza manual ainda não permite a eficiente remoção do biofilme bacteriano ${ }^{22}$.

No que tange as pesquisas em que um maior percentual de CPI foi encontrado em crianças com 4 $\operatorname{anos}^{2,19,25}$, sugere-se que nessa idade as crianças permanecem com lesões de cárie que ainda não tratadas. Aos 5 anos, em contrapartida, e ao se considerar a evolução da doença, observar-se-á uma maior procura aos serviços de tratamento odontológico, provavelmente em decorrência da dor ocasionada pelo agravamento do quadro.

Alguns estudos relatam uma prepoderância de crianças do gênero masculino para o desenvolvimento de lesões de cárie ${ }^{9,28,39}$. Nesse sentido, os mesmos estudos sugerem que meninos possuam um temperamento mais agressivo, oferecendo maior resistência durante a escovação, o que pode levar a alguns cuidadores a desistirem de realizar a higiene bucal de seus filhos, ou ainda considerarem que, culturalmente, os meninos possuem menos atenção a aspectos relacionados à saúde pessoal. Não obstante, na maioria dos estudos não é possível observar tal relação $0^{3,9,17,20,23}$, corroborando o fato de que a cárie não apresenta predileção genética por sexo.

Em se tratando de crianças residentes na zona rural, como grupo populacional de maior risco para desenvolvimento de CPI em relação àquelas que residem nos centros urbanos ${ }^{24,19,20,25}$, argumenta-se o fato de que o distanciamento das cidades pode promover uma restrição no acesso aos serviços de saúde e inadequado e restrito conhecimento sobre os cuidados de saúde bucal e práticas de higiene entre as crianças residentes em áreas rurais ${ }^{24}$.

A maioria dos estudos identifica a baixa renda familiar como um fator fortemente associado à cárie dentária em crianças ${ }^{20,22,24,27,29-31}$. Compreende-se que a baixa renda por si só não determina o desenvolvimento da CPI, mas sim, as repercussões de tal fato que podem elevar o risco de cárie dentária de várias maneiras, a partir da ideia de que as prioridades familiares passam a serem outras, que incluem a subsistência básica da família ${ }^{20,22}$

O grau de escolaridade materna constitui importante fator predisponente para a CPI, uma vez que as mães - aqui consideradas como o cuidador principal - exercem uma grande influência sobre a saúde bucal dos seus filhos, a partir da introdução de hábitos alimentares saudáveis e de hábitos de higiene adequados ${ }^{19,27,29}$. Assim, a falta de informação, comum a pessoas com baixo nível de escolaridade, pode vir a potencializar negativamente a 
condição de saúde bucal da criança.

Frente ao acesso aos serviços de saúde bucal, considera-se que as crianças que são conduzidas desde cedo ao serviço odontológico participam muito mais de uma abordagem preventiva do que curativista, o que reduz a incidência de $\mathrm{CPI}^{30,26,28}$. O estudo de Kuriakose et al. ${ }^{28}$ observou uma relação entre um maior índice de CPI e visitas mais frequentes ao dentista, tal fato se justifica porque se observa que a procura por serviços odontológicos para crianças, em sua maioria, só ocorre quando existem problemas dentários que geram dor ou desconforto, já que a grande maioria da população não tem adequada formação em saúde bucal e detém de limitado acesso ao atendimento preventivo dos serviços públicos de saúde ${ }^{39}$.

Aspectos relacionados à dieta infantil também merecem ser destacados, dentre esses, a amamentação. Embora os benefícios da amamentação para a saúde geral das crianças sejam incontestáveis e incluam, em muitos casos, a sobrevivência das crianças, particularmente daquelas em condições desfavoráveis e/ou que nascem com baixo peso ${ }^{40}$, a amamentação superior a 24 meses pode aumentar o risco de desenvolvimento de lesões de cáries, uma vez que em cada episódio de amamentação há um maior tempo de exposição dos dentes aos carboidratos fermentáveis presentes no leite materno ${ }^{32}$.

Assim sendo, dentre as estratégias preventivas na abordagem da cárie precoce da infância, a Organização Mundial da Saúde e do Ministério da Saúde recomendam o aleitamento materno exclusivo nos primeiros seis meses e a amamentação complementada por outros alimentos por até os dois anos ou mais ${ }^{40}$, idade em que costuma ocorrer o desmame natural. Contudo, crianças cuja amamentação materna prolonga-se possuem menos chances de serem precocemente apresentadas a uma dieta cariogênica ${ }^{3}$ e acredita-se que a relação casuística encontrada nos estudos entre o aleitamento materno prolongado e a CPI ${ }^{19,24,25,32}$, esteja associada com a ausência de higiene bucal adequada após a amamentação, o atraso no início das práticas de higiene bucal e uma falta de assistência dos cuidadores durante a escovação dos dentes.

No que se refere ao aleitamento noturno como um dos principais responsáveis pela ocorrência de CPI ${ }^{19,20,23,25,29,40}$, acredita-se que este achado possa ser atribuído ao fato de que há menos movimentos de autolimpeza e fluxo salivar durante a noite e, portanto, menos capacidade de neutralização dos ácidos. Isto provoca a estagnação do leite na boca durante períodos mais longos de tempo e exposição prolongada dos dentes aos carboidratos fermentáveis ${ }^{19,29}$. Esta constatação apoia a declaração da AAPD segundo a qual a amamentação noturna em livre demanda deve ser evitada após a erupção dentária ${ }^{19}$.

Há também consistentes associações entre a CPI e o uso de chupetas com açúcar ou mel, bem como com o consumo de alimentos e bebidas açucaradas. Devido à praticidade dos chamados "lanches rápidos" - tipicamente ricos em açúcar - observa-se um aumento da oferta de tais lanches na dieta das crianças. Os carboidratros fermentáveis, tais como sacarose e glucose, fornecem os meios ideiais para proliferação das bactérias cariogênicas. Assim, o aumento da frequência de consumo de açúcar aumenta o risco de desmineralização do esmalte e diminui o tempo para remineralização pela saliva que favorecem o aparecimento de lesões de cárie ${ }^{29}$. Como consequência, sugere-se que a exposição precoce ao açúcar repercute na preferência das crianças por doces, fazendo com que, cada vez mais, haja o consumo de alimentos adoçados ${ }^{22,34,35}$

Ademais, cuidadores que reconhecem a importância de hábitos saudáveis de higiene bucal exercem um dos principais papéis preventivos para a inibição dos índices de cárie dentária em crianças. São estes os responsáveis pelo início das escovações, de hábitos de dieta saudáveis e pelas visitas aos dentistas, dentre outros ${ }^{2}$. A literatura afirma que as crianças com até 6 anos de idade, não entendem a importância da escovação ou não possuem a destreza manual ideal para realização de uma efetiva remoção do biofilme dentário ${ }^{22}$. Destarte, a assistência e a orientação dos cuidadores são essenciais para redução dos riscos de desenvolvimento da cárie.

A literatura defende que a escovação dos dentes das crianças deve ser instituída logo quando da erupção do primeiro dente, já que os dados apontam que crianças que começaram a escovar os dentes tardiamente apresentaram maior prevalência de cárie precoce na infância ${ }^{19,22,26,34}$.

Em se tratando da frequência de escovação, acreditase que ao se realizar mais que uma escovação diária há maiores chances de se atingir áreas não alcançadas com uma única escovação, promovendo, portanto, com um maior benefício na prevenção da $\mathrm{CPI}^{2,19,23,28,29}$. Eleutério et al. ${ }^{39}$ verificaram, no entanto, uma relação conflitante entre a frequência de escovação e presença de CPI. Contudo, é válido considerar que os cuidadores entrevistados em tal pesquisa podem ter alterado a frequência real de escovação, por ser amplamente divulgado que a escovação deve ser realizada, pelo menos, três vezes ao dia.

Crianças que usam dentifrício fluoretado possuem prevalência de cárie significativamente mais baixa. A escovação com dentifrício fluoretado tem desempenhado um papel importante no declínio de cárie, porque o íon flúor se precipita frente ao desafio cariogênico, não permitindo a desmineralização das estruturas dentais. No entanto, o impacto da utilização do dentifrício fluoretado e da ingestão total de flúor em crianças é uma questão controversa, pelo risco de fluorose. A fluorose tem sido associada com a ingestão de flúor durante o desenvolvimento do esmalte, com sua gravidade dependente da dose, da duração e do tempo de ingestão ${ }^{22}$.

O uso racional de fluoreto vem sendo considerado instrumento eficaz e seguro na prevenção e controle da cárie dentária, apresentando-se como um importante elemento estratégico nos sistemas de prevenção da cárie dentária quando corretamente aplicado, independentemente da forma como se dá o contato tópico. A AAPD ${ }^{1}$ sugere o uso de dentifrício fluoretado (na concentração de 1.000 ppm F) duas vezes por dia desde a erupção do primeiro dente decíduo. Os cuidadores devem ser orientados sobre o risco de cárie do seu filho, dispensando um volume adequado de pasta de dente a cada idade, escova de tamanho adequado e auxiliando na escovação das crianças. Em crianças com menos de 2 anos de idade, a quantidade de dentifrício fluoretado recomendado é a de um grão de arroz, e em crianças de 2 a 5 anos, a quantidade deve ser semelhante ao tamanho de uma ervilha.

Observa-se, também, uma associação entre a CPI e as condições crônicas de saúde encontradas nos estudos, a qual pode ser parcialmente atribuída à utilização de medicamentos para o tratamento desses pacientes, a alterações na composição da saliva e do fluxo salivar, um consumo maior de doce ou ainda pelo fato de que os cuidadores de crianças com doenças crônicas, muitas vezes, 
superestimam a condição médica da criança dando pouca importância aos procedimentos de higiene bucal ${ }^{15}$.

\section{CONCLUSÃO}

Considerando que todos os fatores de risco se encontram em íntima relação, não é possivel avaliar quais, dentre os fatores associados, exercem maior ou menor influência no surgimento da cárie precoce da infância. Não obstante, alguns fatores de risco foram prepoderantes na literatura consultada, dentre os quais: crianças mais velhas; baixo nível de escolaridade dos principais cuidadores; baixa renda familiar; habitantes da zona rural; escasso acesso a serviços de saúde bucal; hábitos alimentares inadequados, com destaque para amamentação noturna e consumo de alimentos e bebidas com sabor doce entre as principais refeições; conhecimento limitado dos cuidadores sobre higiene bucal, especialmente em relação à idade de início da escovação e a responsabilidade destes nas escovações; além do comprometimento sistêmico da criança.

Destarte, o conhecimento sobre os fatores de risco associados ao surgimento e a progressão da cárie precoce da infância, permite uma atuação odontológica centrada na prevenção dos agravos à saúde bucal. Nesse sentido, estratégias preventivas específicas para cada grupo de risco devem ser elaboradas, de modo que as intervenções sejam efetivas no âmbito da promoção de saúde.

\section{REFERÊNCIAS}

1. American Academy of Pediatric Dentistry (AAPD). Policy on Early Childhood Caries (ECC): Classifications, Consequences, and Preventive Strategies. Pediatr Dent. 2014; 36(6):50-2.

2. Folayan MO, Kolawole KA, Oziegbe EO, Oyedele T, Oshomoji OV, Chukwumah NM et al. Prevalence, and early childhood caries risk indicators in preschool children in suburban Nigeria. BMC Oral Health. 2015; 15(72):1-12.

3. Majorana A, Cagetti MG, Bardellini E, Amadoril F, Conti G, Strohmenger L et al. Feeding and smoking habits as cumulative risk factors for early childhood caries in toddlers, after adjustment for several behavioral determinants: a retrospective study. BMC Pediatrics, 2014; 14(45):1-8.

4. Scalioni FAR, Figueiredo SR, Curcio WB, Alves RT, Leite ICG, Ribeiro RA. Hábitos de Dieta e Cárie Precoce da Infância em Crianças Atendidas em Faculdade de Odontologia Brasileira. Pesq Bras Odontoped Clin Integr. 2012;12(3):399-404.

5. Losso EM, Tavares MCR, Silva JYB, Urban CA. Severe early childhood caries: an integral approach. J Pediatr. 2009; 85(4):295-300.

6. Brasil. SB Brasil 2010: Pesquisa Nacional de Saúde Bucal: resultados principais. Ministério da Saúde. Secretaria de Atenção à Saúde. Secretaria de Vigilância em Saúde. Brasília: Ministério da Saúde, 2012.

7. Alkarimi HA, Watt RG, Pikhart H, Jawadi AH, Sheiham A, Tsakos G. Impact of treating dental caries on schoolchildren's anthropometric, dental, satisfaction and appetite outcomes: a randomized controlled trial. BMC Public Health. 2012; 12(706):1-8.

8. Dujister D, Sheiham A, Hobdell MH, Itchon G, Monse B. Associations between oral health-related impacts and rate of weight gain after extraction of pulpally involved teeth in underweight preschool Filipino children. BMC Public Health. 2013; 13(533):1-9.
9. Schroth RJ, Halchuk S, Star L. Prevalence and risk factors of caregiver reported Severe Early Childhood Caries in Manitoba First Nations children: results from the RHS Phase 2 (2008-2010). Int J Circumpolar Health. 2013; 72(21167):1-10

10. Clementino MA, Gomes MC, Pinto-Sarmento TCA, Martins CC, Granville-Garcia AF, Paiva SM. Perceived Impact of Dental Pain on the Quality of Life of Preschool Children and Their Families. PLoS One. 2015; 10(6):1-13.

11. Gomes AC, Pinto-Sarmento TCA, Costa EMMB, Martins CC, Granville-Garcia, AF, Paiva SM. Impact of oral health conditions on the quality of life of preschool children and their families: a cross-sectional study. Health Qual Life Outcomes. 2014; 12(55):1-12.

12. Boing AF, Bastos JL, Peres KG, Antunes JLF, Peres MA. Determinantes sociais da saúde e cárie dentária no Brasil: revisão sistemática da literatura no período de 1999 a 2010. Rev Bras Epidemiol. 2014; 17(Suppl2):102-15.

13. Marsh PD. Microbial ecology of dental plaque and its significance in health and disease. Adv Dent Res. 1994; 8(2):263-71.

14. Takahashi N, Nyvad B. Caries ecology revisited: microbial dynamics and the caries process. Caries Res. 2008; 42(6):409-18.

15. Echeverría SL, Herrera GO, Henriquez D'A E, Sepulveda RR, Maldonado LP. Prevalencia de caries temprana de la infancia en niños con enfermedades respiratorias crónicas. Rev Chil Pediatr. 2012; 83(6):563-9

16. Narvai PC. Cárie dentária e flúor: uma relação do século XX. Ciênc. saúde colet. 2000; 5(2):381-92.

17. Cardenas CF, Perona GMP. Factores de riesgo asociados a la prevalencia de caries de aparición temprana en niños de 1 a 3 años en una población peruana. Odontol Pediatr. 2013;12(2):110-8.

18. Lima JEO. Cárie dentária: um novo conceito. R Dental Press Ortodon Ortop Facial. 2007; 12(6):119-30.

19. Jain M, Namdey R, Bodh M, Dutta S, Singhal P, Kumar A. Social and Behavioral Determinants for Early Childhood Caries among Preschool Children in India. J Dent Res Dent Clin Dent Prospect. 2015; 9(2):115-20.

20. Wulaerhan J, Abudureyimu A, Bao XL, Zhao J. Risk determinants associated with early childhood caries in Uygur children: a preschool-based cross-sectional study. BMC Oral Health. 2014; 14(136):1-8.

21. Abbasoglu Z, Tanboga I, Küchler EC, Deeley K, Weber $\mathrm{M}$, Kaspar C, et al. Early childhood caries is associated with genetic variants in enamel formation and immune response genes. Caries Res. 2015; 49(1):70-7.

22. Prakash P, Subramaniam P, Durgesh BH, Konde S. Prevalence of early childhood caries and associated risk factors in preschool children of urban Bangalore, India: A cross-sectional study. Eur J Dent. 2012; 6(2):141-52.

23. Congiu G, Campus G, Sale S, Spano G, Cagetti MG, Luglie PF. Early childhood caries and associated determinants: a cross-sectional study on Italian preschool children. J Public Health Dent. 2014; 74(2):147-52.

24. Correa-Faria P, Martins-Junior PA, Andrade RGV, Marques LS, Ramos-Jorge ML. Factors associated with the development of early childhood caries among Brazilian preschoolers. Braz Oral Res. 2013;27(4):35662. 
25. Sanchez CZ, Toledo PP, Caceres JJO. Prevalencia de caries temprana de la infancia y sus factores asociados en niños chilenos de 2 y 4 años. Int J Odontostomatol, 2011; 5(2):171-7.

26. Ghazal T, Levy SMM, Childers NK, Broffitt B, Cutter GR, Wiener HW et al. Factors associated with early childhood caries incidence among high caries-risk children. Community Dent Oral Epidemiol. 2015; 43(4):366-74.

27. Kato T, Yorifuji T, Yamakawa M, Inoue S, Saito K, Doi $\mathrm{H}$ et al. Association of breast feeding with early childhood dental caries: Japanese population-based study. BMJ Open. 2015; 5(3):1-9.

28. Kuriakose S, Prasannan M, Remya KC, Kurian J, Sreejith KR. Prevalence of early childhood caries among preschool children in Trivandrum and its association with various risk factors. Contemp Clin Dent. 2015; 6(1):69-73.

29. Peltzer K, Mongkolchati, A. Severe early childhood caries and social determinants in three-year-old children from Northern Thailand: a birth cohort study. BMC Oral Health, 2015; 15(108):1-7

30. Darmawikarta D, Chen Y, Carsley S, Birken CS, Parkin PC, Schroth RJ, Maguire JL. Factors Associated With Dental Care Utilization in Early Childhood. Pediatrics. 2014; 133(6):1594-600.

31. Moimaz SAS, Fadel CB, Lolli LF, Garbin CAS, Garbin AJI, Saliba NA. Social aspects of dental caries in the context of mother-child pairs. J Appl Oral Sci. 2014; 22(1):73-8.

32. Chaffee BW, Feldens CA, Vitolo MR. Association of long-duration breastfeeding and dental caries estimated with marginal structural models. Ann Epidemiol. 2014; 24(6):448-54.

33. Ozen B, Van-Strijp AJ, Ozer L, Olmus H, Genc A, Cehreli SB. Evaluation of Possible Associated Factors for Early Childhood Caries and Severe Early Childhood Caries: A Multicenter Cross-Sectional Survey. J Clin Pediatr Dent. 2016; 40(2):118-23.

34. Nunes AM, Da Silva AA, Alves CM, Hugo FN, Ribeiro CC. Factors underlying the polarization of early childhood caries within a high-risk population. BMC Public Health. 2014; 14(988):1-9

35. Yokomichi H, Tanaka T, Suzuki K, Akiyama T, Yamagata Z. Macrosomic Neonates Carry Increased Risk of Dental Caries in Early Childhood: Findings from a Cohort Study, the Okinawa Child Health Study, Japan. PLoS One. 2015; 10(7):1-12

36. Salama F, Abobakr I, Al-Khodair N, Al-Wakeel M. Comparison of Saudi child versus parent-report of child tooth-brushing practices. Niger J Clin Pract. 2016 JulAug;19(4):449-54.

37. Marinho VCC, Chong LY, Worthington HV, Walsh, T. Fluoride mouthrinses for preventing dental caries in children and adolescents. Cochrane Database of Systematic Reviews. 2016; 7:1-135.

38. Santos Junior VE, Sousa RMB, Oliveira MC, Junior AFC, Rosenblatt A. Early childhood caries and its relationship with perinatal, socioeconomic and nutritional risks: a cross-sectional study. BMC Oral Health. 2014; 14(47):1-5.

39. Eleuterio ASL, Cota ALS, Kobayashi TY, Silva SMB. Avaliação clínica da saúde bucal de crianças dos municípios de Alfenas e Areado, Minas Gerais, Brasil. Pesqui Bras Odontopediatria Clín Integr. 2012; 12(2):195-201.
40. Brasil. Saúde da criança: nutrição infantil: aleitamento materno e alimentação complementar. Ministério da Saúde. Secretaria de Atenção à Saúde. Departamento de Atenção Básica. Brasília: Ministério da Saúde, 2009.

\section{CONFLITO DE INTERESSES}

Os autores declaram não haver conflitos de interesse.

\section{AUTOR PARA CORRESPONDÊNCIA}

Maria das Graças Barbosa da Silva

mgalbarbosaesilva@gmail.com

Submetido em 27/09/2017 Aceito em 02/11/2017 